\title{
Kinematics and Injury Analysis of Front and Rear Child Pillion Passenger in Motorcycle Crash
}

\author{
S. Koetniyom ${ }^{*}$, J. Carmai ${ }^{1}$, K. A. A. Kassim ${ }^{2}$ and Y. Ahmad ${ }^{2}$ \\ ${ }^{1}$ Automotive Safety and Assessment Engineering Research Centre \\ King Mongkut's University of Technology North Bangkok \\ 1518 Pracharat 1 Road,Wongsawang, Bangsue, Bangkok 10800 Thailand \\ *Email: saiprasit.k@tggs.kmutnb.ac.th \\ Phone: +6625552000; Fax: +6625874350 \\ ${ }^{2}$ Malaysia Institute of Road Safety Research, \\ 125-135 Jalan TKS 1, Taman Kajang Sentral, 43000 Kajang, Selangor, Malaysia
}

\begin{abstract}
The purpose of this work is to study the kinematics and injury of child pillion passenger from motorcycle-to-car crash tests. Two crash tests for rear and front child pillions were set up. The kinematics of dummies were analysed from accelerometer data and high speed camera pictures. The kinematics and injury mechanisms of the child passenger from both tests are significantly different. For the rear child pillion test, the rider impacted the car before the child passenger. Both rider and child were ejected upward. The child's head motion was curvilinear towards the car structure. This results in severe head injury due to high HIC. The child sitting at front translated in the longitudinal axis of the motorcycle and impacted the car before the rider. The child's torso strongly hit to the handlebar first then head hit the car. This results in low value of HIC. The child's upper-body including neck were compressed between the car and the rider's torso leading to high risk of severe thorax and neck injuries. The results reveal that the child sitting behind the rider has higher risk of severe head injury while the child sitting before the rider has higher risk of thorax and neck injuries.
\end{abstract}

Keywords: Child pillion passenger; motorcycle crash test; head injury criteria; kinematics; injury mechanism.

\section{INTRODUCTION}

Motorcycle is the most unsafe form of transportation and often involve in large number of annual fatalities. From the journal articles of medical related motorcycle and technical reports of the US National Highway Traffic Safety Administration, the risk factors of motorcycle accident are composed of helmet effectiveness, alcohol drinking motorcycle riders, inexperience of driver training, conspicuity of motorcycle, license of ownership, riding speed and risk-taking behavior [1]. According to global road safety report by WHO the number of deaths by motorcycle in Southeast-Asia in road accidents (34\%) is the largest as compared to car accidents (16\%) [2]. Therefore, motorcycle safety is of prime importance in Southeast-Asian regions like Thailand due to large number of motorcycle users. In developed countries, passive safety research in motorcycle focuses on the impact tests against vehicle, guard rails or barriers to investigate the protection of safety parts in motorcycle and on riders [3] [4]. 
In low and middle-income countries, child pillion passengers are very common in motorcycles which are relatively unsafe vehicle with unprotected users. From WHO published data in 2015, injury proportion of child pillion in motorcycle are $17.2 \%$, $24.6 \%$ and $61.9 \%$ for India, Nepal and Thailand respectively. There is $37.2 \%$ of child pillion passengers from India and Thailand in which children are subjected to head and neck injuries [5]. In Malaysia, a total 915 school children representing $10 \%$ of the whole selected schools' populations are child pillion passengers in motorcycles for school transportation. Safety helmet use for child pillion passengers was $14.7 \%$ [6]. However, children are most likely to have severe impact with hitting objects and thus have less survival chances if injured in case of motorcycle crash event. From the national police report crash database between 2007 and 2011, $18.8 \%$ of child pillion passengers are subjected to head injuries in Malaysia [7]. Therefore, there are some recommendations to improve the child pillion passengers in motorcycle such as biomechanical research for appropriate helmet, child seat and infant sling [5]. In order to improve countermeasures, understanding of kinematics and injury mechanisms of child pillion passenger during impact is necessary. Motorcycle crash tests can give insight into kinematics and injury sustained by riders and their passengers. Laboratorybased motorcycle crash tests [8] or full-scale crash tests [3] [9] of standard type of motorcycles were conducted. However, they usually test with the rider only.

This work, therefore, would like to investigate the most fatal case of child injuries by conducting two full-scale small motorcycle crash tests. They were performed in collaboration between King Mongkut's University of Technology North Bangkok (KMUTNB) and ASEAN New Car Assessment Protocol (NCAP) at Malaysian Institute of Road Safety (MIROS), PC3 Crash Test Laboratory, Melaka Malaysia. The objective of these crash tests is to study the kinematics and head injuries of front and rear child pillion passengers. In this study, crash test procedure is based on International Organization for Standardization (ISO 13232-6:2005 part 6), full-scale impact-test procedures for research evaluation of rider crash protective devices fitted to motorcycles together with the most common scenario accidents in Thailand [10]. In addition, the most popular motorcycle riding patterns for child pillion passengers in middle-income countries and densely populated areas are the rear and front positions of motorcycle rider. Thus, the setup, configuration and procedures of crash test are developed to investigate kinematics and injury of child in both most common motorcycle riding patterns for child pillion passengers.

\section{VEHICLE CRASH TEST STANDARD AND INJURY CRITERIA}

Based on the standard ISO 13232, the complete test and analysis procedures for research evaluation of rider crash protective devices fitted to motorcycles are introduced. The full standard ISO 13232 consists of impact conditions, Motorcycle Anthropometric Test Device (MATD) dummy, instrumentation, variable measurements, injury indices and impact test procedures for computer FE simulations in motorcycle. In this experiment, part 6 of ISO 13232-Full-scale impact test of motorcycle are composed of dummies preparation, installation procedures, test setup configurations, camera locations and the various types of test conditions [11]. In type 4 of test condition, the Motorcycle (MC) collides with the side of Opposite Vehicle (OV) at center of its door with 90 degrees impact angle. In this condition, the $\mathrm{OV}$ is at rest and the MC speed is at $13.4 \mathrm{~m} / \mathrm{s}(48.2 \mathrm{~km} / \mathrm{h})$. 
In vehicle crash test, Head Injury Criterion (HIC) is normally used to evaluate the vehicle safety performance in case of human head impact with objects. HIC is calculated from the accelerometer data from the head of the dummy and is represented by the following equation.

$$
H I C=\left[\left(t_{2}-t_{1}\right)\left\{\frac{1}{t_{2}-t_{1}} \int_{t_{1}}^{t_{2}} a(t) d t\right\}^{2.5}\right]
$$

Where acceleration with respect to time " $a(t)$ " is obtained from the accelerometer in "g" (standard gravity acceleration) and calculated in the maximum standard time interval $\left(t_{2}-t_{1}\right)$ of 15 and 36 ms. With regard to the protection of the occupants in event of a frontal collision from United Nations Economic Commission for Europe (UNECE or ECE) Regulation No. 94, the test protocol uses $36 \mathrm{~ms}$ standard time interval for determination of HIC, as the duration of impact is longer in this case [12]. This is due to airbag and other protective restrained devices involved in frontal impact tests. The requirement limits of $\mathrm{HIC}$ for the protection of occupants in frontal collision shall not exceed 1000. In addition, Euro NCAP Pedestrian Protection Tests Protocol uses only 15 ms because the impact time is relatively short due to quick impact of pedestrian head with objects without any restraints [13]. Therefore, the threshold of the upper performance limit for the HIC is 650 [13].

\section{METHODOLOGY}

\section{Vehicle preparation}

For two crash tests, the selected MC was Yamaha Lagenda 115Z Malaysia model [14]. The OV was Perodua Myvi Model 2014 with weight of $950 \mathrm{~kg}$ [15]. The MC was prepared for crash test according to ISO 13232-6. Fuel tank was emptied by siphoning out any fuel in the fuel tank. The centerline of seat was marked with a marking tape. The MC was secured in the sled carrier trolley in the upright position with supports from both sides of MC handle bar as shown in Figure 1. The sled carrier function was to keep the MC in upright position, accelerate it and then release it to hit the car under inertial and gravitational effects.

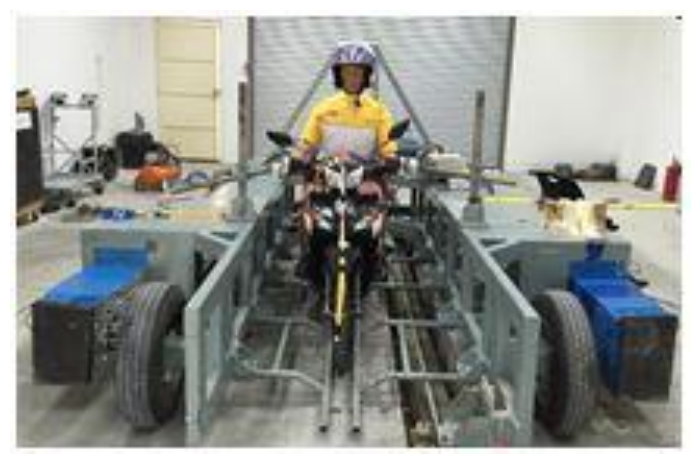

Figure 1. MC sled carrier. 
For the OV, the fuel tank was emptied, and the battery was removed. The transmission of vehicle was kept in neutral. The service brakes were engaged to prevent any movement of car during test. The center of left side of the front door was marked where the MC was supposed to be hit and aligned at the test track with center of MC tire as shown in Figure 2.

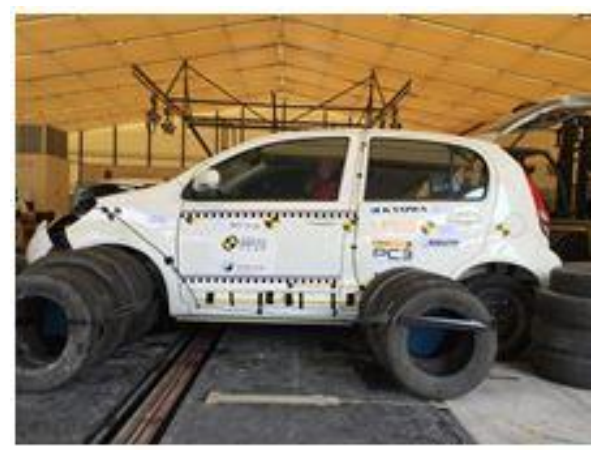

Figure 2. Position of opposing vehicle (OV).

\section{Dummy preparation}

Motorcyclist Anthropometric Test Device (MATD) was used as adult rider and installed on the rider seat of MC. Both hands of MATD were placed on the handle bar and the helmet was placed on MATD head. A P6 child dummy for 6 years-old was used as child pillion with two different seat positions. Therefore, two crash test conditions with the P6 dummy installed at the front as shown in Figure 3(a) and back of the rider as shown in Figure 3(b). In the opposite vehicle, two Hybrid-III dummies were used as a driver and passenger and were placed at front seat of opposing vehicle respectively.

A triaxial model ASDE-A accelerometer is used with a dynamic range of $+/-$ 1000G together with DIS 506A data logger that can be independently recorded up to 12 seconds at $20 \mathrm{kHz}$ from Kyowa Electronics Instruments. The dimensions of accelerometer and data logger which can be installed at the center of gravity of the P6 child dummy are $4.6 \times 4.9 \times 13.5 \mathrm{~mm}$ and $18 \times 26 \times 62 \mathrm{~mm}$ respectively. The installation process of accelerometer and data logger inside the P6 child dummy are illustrated in Figure 4.

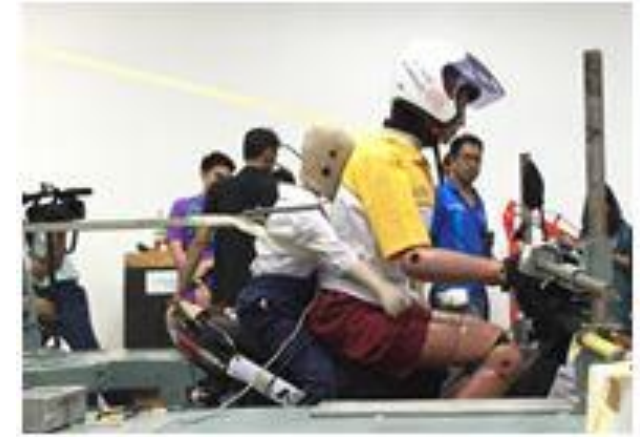

(a)

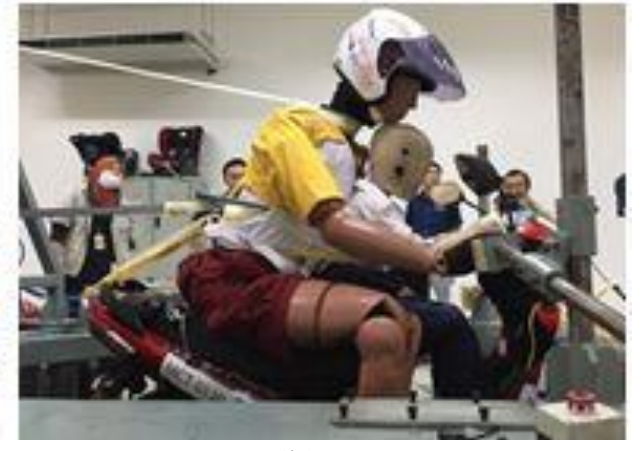

(b)

Figure 3. Child dummy positioning (a) at the back of the MATD (b) at the front of the MATD. 


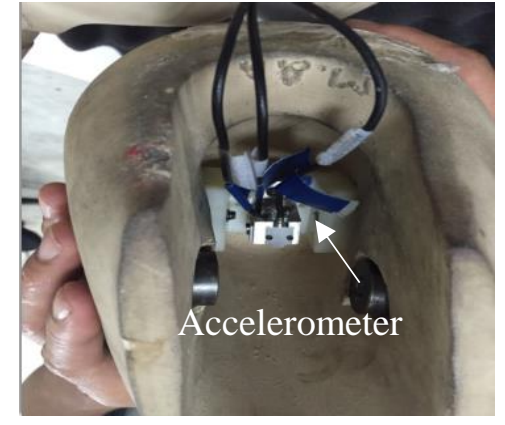

(a)

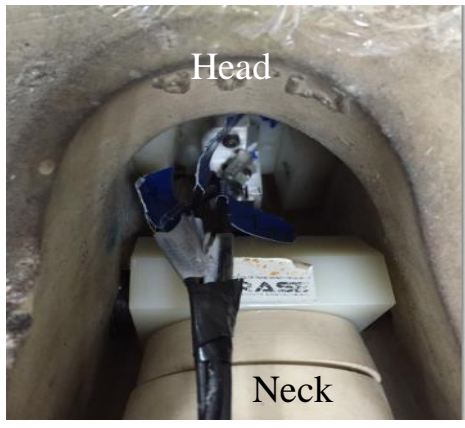

(b)

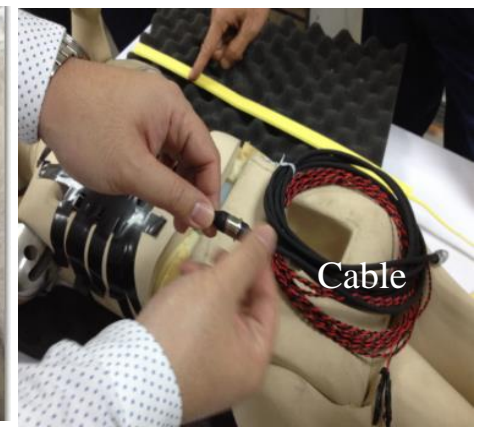

(c)

Figure 4. Installation process of accelerometer and data logger in child dummy (a) attach accelerometer in P6 dummy head (b) assembly the head with the neck (c) connect cable to accelerometer.

\section{Test Procedure}

To capture the kinematics of dummies, high-speed cameras were installed at various locations as illustrated in Figure 5. Number of cameras used and installed positions were the same for both crash tests. Four high-speed cameras were installed at left and right sides of the MC for a wide- and a close-views respectively. One camera right above the roof of the car for a top view and one camera on board of the car as shown in Figure 5.

The crash test configuration was based on ISO 13232-6 Type 4 [11] standard procedure in which the OV was set be stationary with 90 degrees impact angle with the MC. The opposite vehicle was setup according to standard regulations ISO 13232-6 [11] at right angle to the MC. Two tests with following variations were conducted. The code number for two tests are MCT 0119 and MCT 0120 for child dummy at the back and the front positions of the ride respectively.

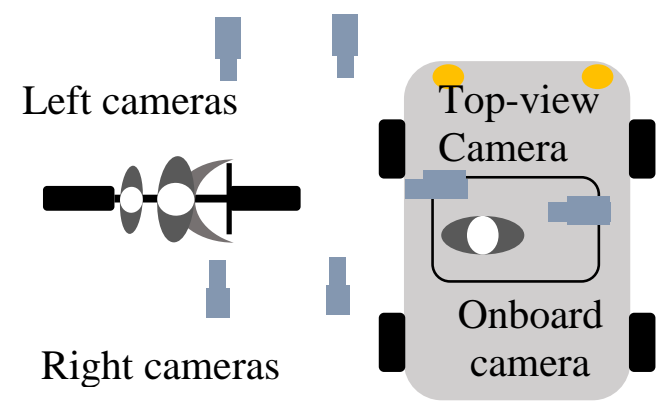

Figure 5. High speed camera installation positions.

Once the equipment was installed and ready for test, an overall check was performed to ensure the compliance of all the rules. The retractor cable connector was attached with the MC sled carrier. Then, the track and other safety checks were inspected. Finally, the connector is attached to the trolley to accelerate the MC to the specified speed. 


\section{EXPERIMENTAL RESULTS}

\section{Rear Child Pillion Test}

The MC impact speed in the first test (child sitting behind the rider) reached up to 42.2 $\mathrm{km} / \mathrm{h}$ measured by a speed sensor before deformable barriers at both sides of trolley hit the stoppers. However, the leg of rider was dragged by the stoppers before the impact. This incident caused the child dummy being thrown and toppled over the MC to hit the car. The child head impact speed of $40.5 \mathrm{~km} / \mathrm{h}$ was calculated using pictures from the top-view high speed camera with 1000 frames per second. The resultant acceleration experienced by head of child dummy in first test is shown in Figure 6. The acceleration graph started from 0 when the MC was released from the sled carrier at time 0 . The first peak of acceleration occurred at $271 \mathrm{~ms}$ with the highest value of $7630.41 \mathrm{~m} / \mathrm{s}^{2}$. This is due to the impact of child's head with the A-Pillar of the car. The calculated $\mathrm{HIC}_{15}$ is as high as 10522.54. During 300-400 ms, another high series of accelerations are observed due to child's head rebound effect. Another smaller peak of acceleration is observed at $1500 \mathrm{~ms}$ due to the second impact with ground.

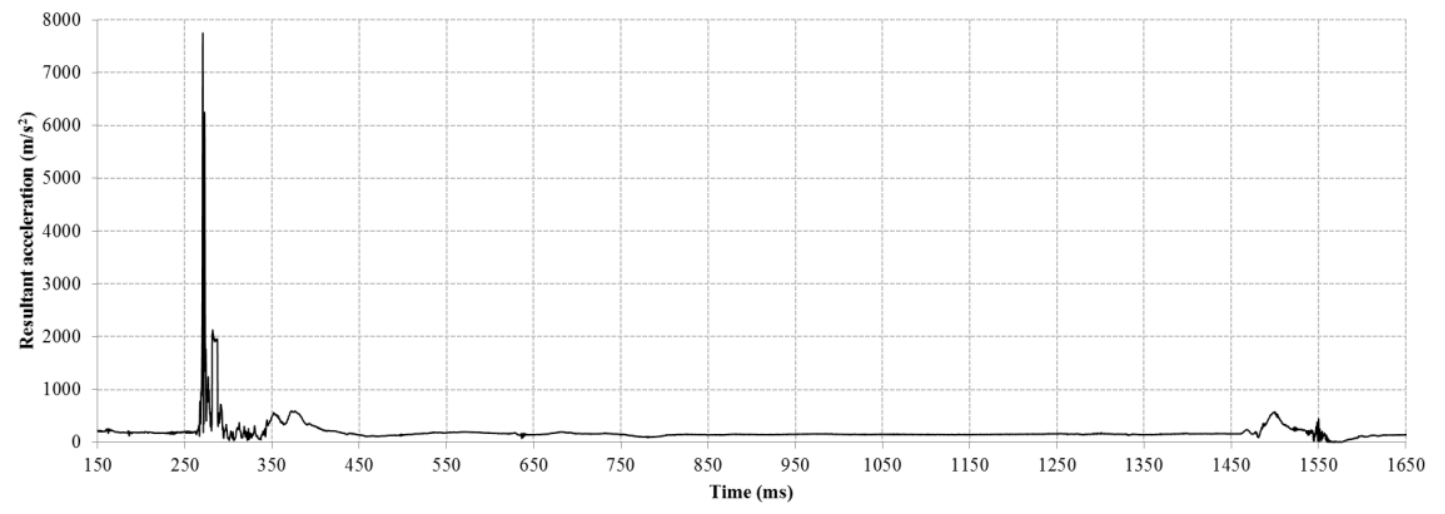

Figure 6. Resultant acceleration data at child head dummy for the first test (MCT0119).

The component accelerations in three axes are plotted in order to observe the impact direction related to the child's head as shown in Figure 7. In addition, the corresponding kinematics of dummies for near-side view at various time steps are shown in Figure 8 (a-d) together with top view at various time step as shown in Figure 8 (e-f). Figure 9 (a-e) illustrates the kinematics for far-side view. The acceleration in the negative X-direction at $271-272 \mathrm{~ms}$ is observed due to inertial effects indicating deceleration in backward direction. The second peak is observed in Z-direction at 272$273 \mathrm{~ms}$ because the moving body's weight exerted force on the head which impacted at downside of A-pillar as indicated in Figure 8 at $272 \mathrm{~ms}$. Since Y-axis is in sideward direction, the graph remains constant which shows zero sideward acceleration. The body of the dummy was thrown with high forces which lead to rotatory motion of head after impact to A-pillar as evident in Figure 8 at $281 \mathrm{~ms}$. The negative deceleration of head in X-direction during 281 to $288 \mathrm{~ms}$ in Figure 7 also confirms this kinematics. 


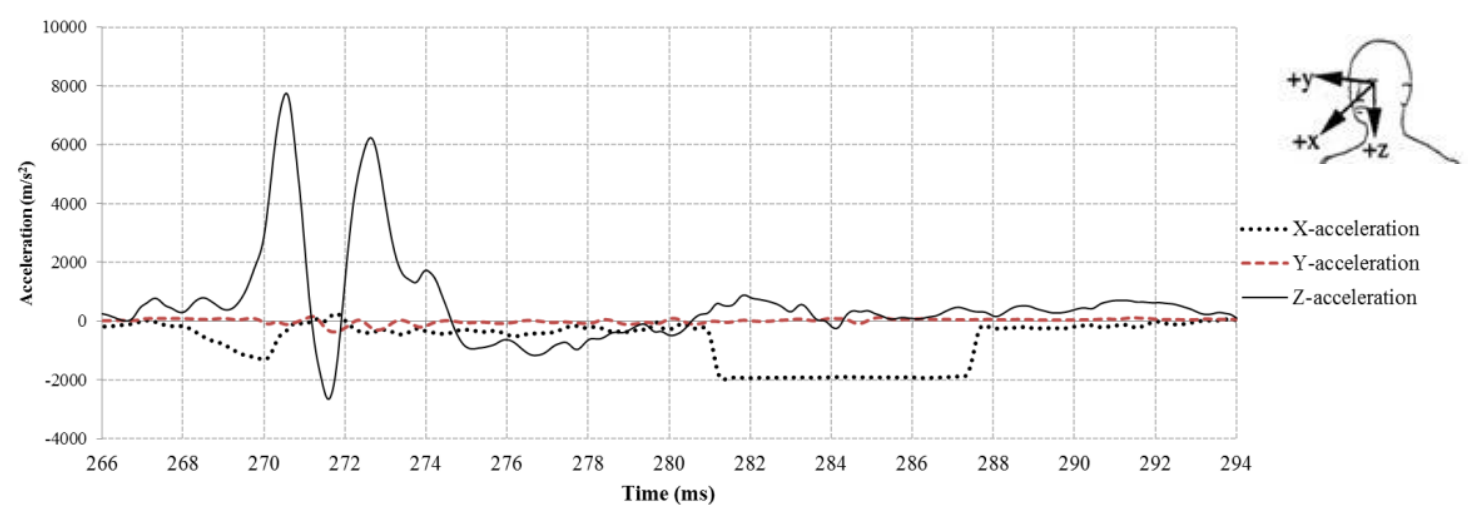

Figure 7. Component acceleration data of child's dummy head at 266-294 ms (MCT0119).

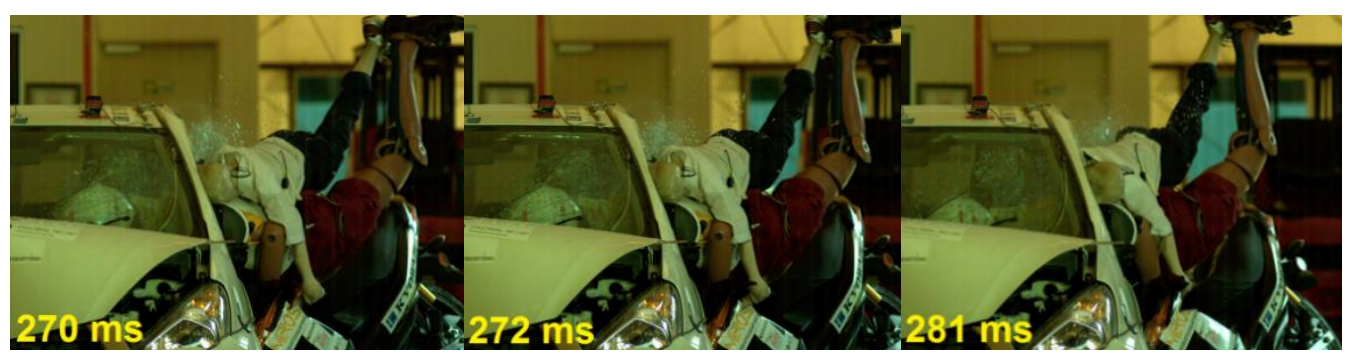

(a)

(b)

(c)

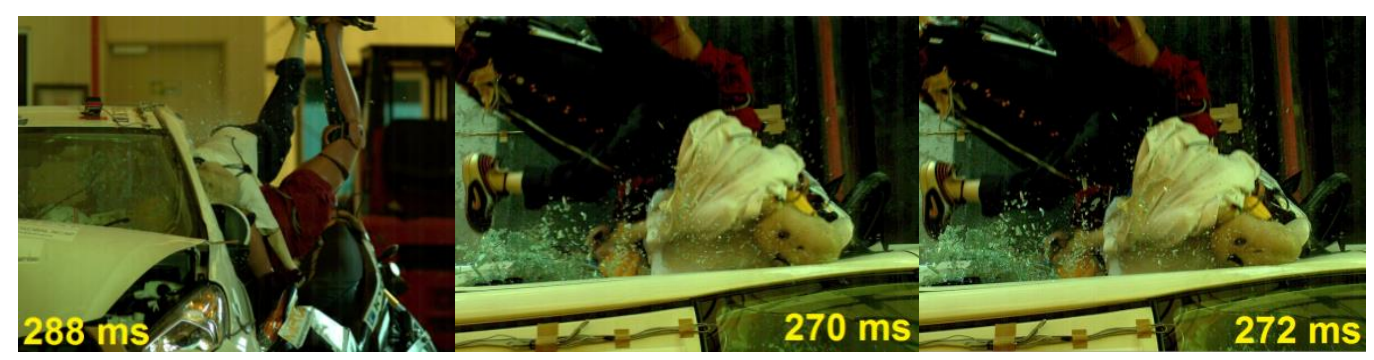

(d)

(e)

(f)

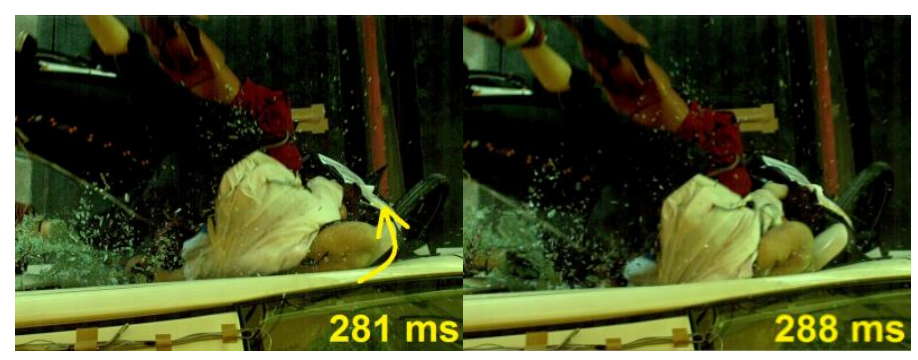

(g)

(h)

Figure 8. Kinematics of dummies )MCT0119( for near-side view at (a) $270 \mathrm{~ms}$, (b) 272 ms, (c) $281 \mathrm{~ms}$, (d) $288 \mathrm{~ms}$, and for top view at (e) $270 \mathrm{~ms}$, (f) $272 \mathrm{~ms}$, (g) $281 \mathrm{~ms}$ and; (h) $288 \mathrm{~ms}$. 


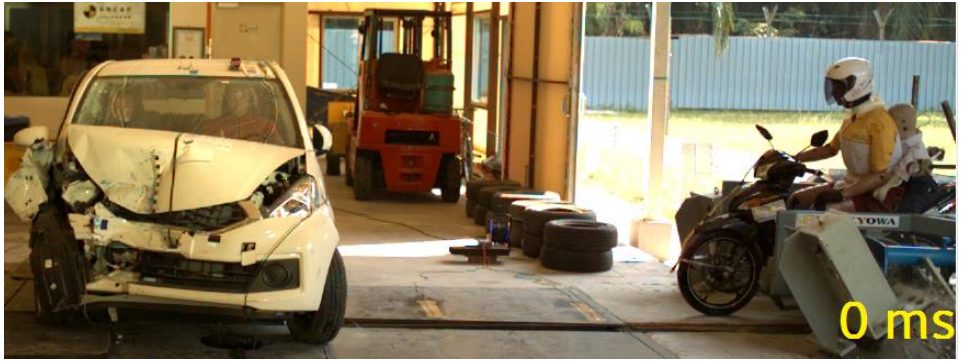

(a)

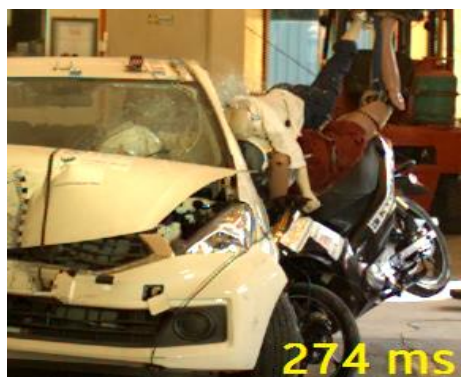

(c)

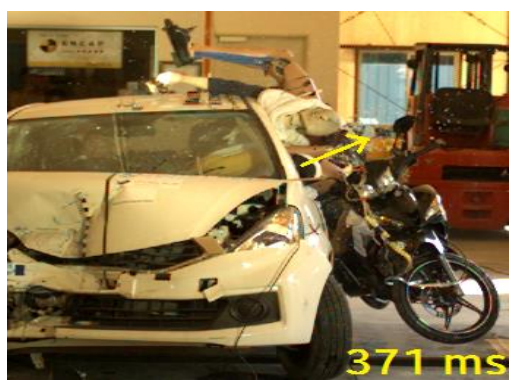

(d)

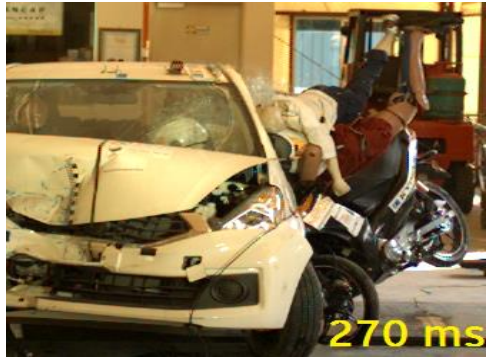

(b)

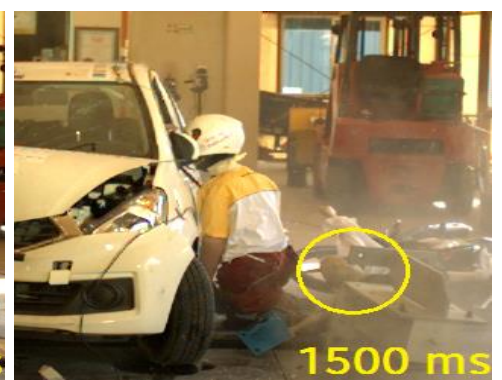

(e)

Figure 9. Kinematics of dummies )MCT0119( from far-side view at (a) $0 \mathrm{~ms}$, (b) 270 ms, (c) $274 \mathrm{~ms}$, (d) $371 \mathrm{~ms}$ and; (e) $1500 \mathrm{~ms}$.

\section{Front Child Pillion Test}

The MC impact speed in the second test (child sitting before the rider) reached up to $27.2 \mathrm{~km} / \mathrm{h}$ measured by the speed sensor. Both child passenger and the rider translated forward together. The child's torso hit the handle bar first. The child's head impacted with speed of $22.2 \mathrm{~km} / \mathrm{h}$ was calculated from the high speed camera with 1000 frames per second.

The resultant acceleration at the head of child dummy during the impact is shown in Figure 10. It reveals that the high acceleration peaks are observed between 200-300 ms when the child's head hit the side mirror first and then the side of car. At $215 \mathrm{~ms}$, the child's head experienced the first peak of acceleration due to the $\mathrm{Z}$ component of acceleration as shown in Figure 11. Figure 12 (a) illustrates kinematics of dummies before impact at time 0 . Kinematics of dummies for near-side view at various time steps are also shown in Figure 12 (b-f) together with top view at various time step as shown in Figure $12(\mathrm{~g}-\mathrm{k})$. This is because the head hit the downside of side mirror resulting in the high acceleration. When the head dragged along the stiff part of mirror at $222 \mathrm{~ms}$, the second peak of acceleration in the $\mathrm{Z}$ direction is observed. At $227 \mathrm{~ms}$, the child's head against the mirror produced the deceleration in the backward X direction (negative acceleration). After $245 \mathrm{~ms}$, the head was subjected to the second impact from the rider body which led to the acceleration in the forward X-direction (positive acceleration). While the rider body hit the head of child dummy, the highest peak acceleration in the $\mathrm{Z}$ direction was at $257 \mathrm{~ms}$. After $265 \mathrm{~ms}$, the child head and rider body moved together resulting in the deceleration in the backward $\mathrm{X}$ direction. However, HIC $_{15}$ value of child head dummy is only 377.29 because of low resultant acceleration. After the MC hit the car, adult rider compressed the child dummy against the lateral side of the car with mass of both dummies as shown in Figure 12 at time 273 ms. 


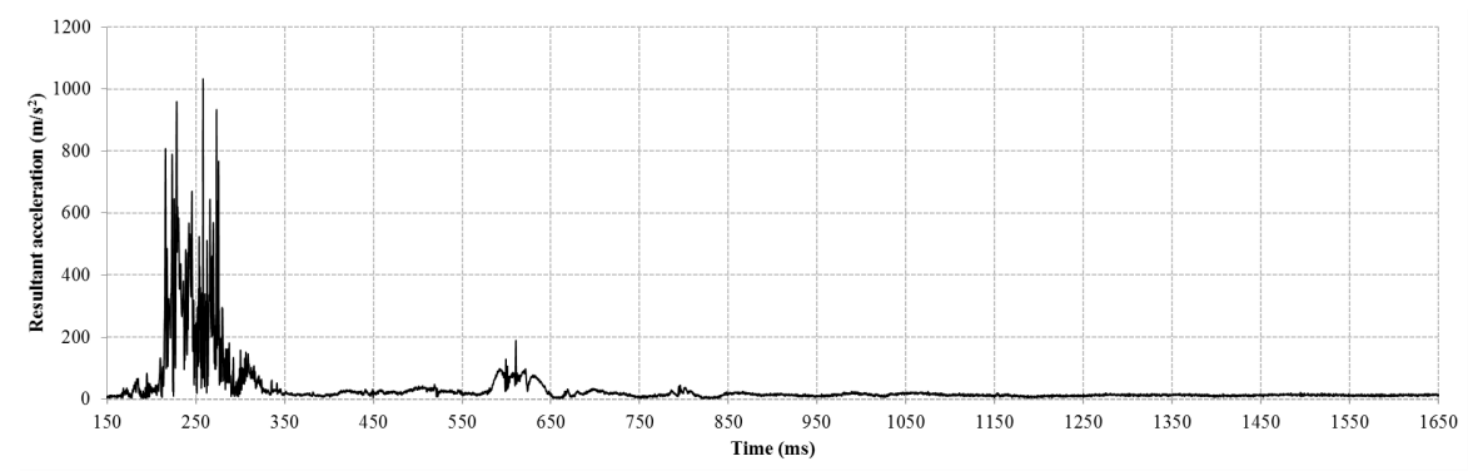

Figure 10. Resultant acceleration data at child's head for the second test (MCT0120).

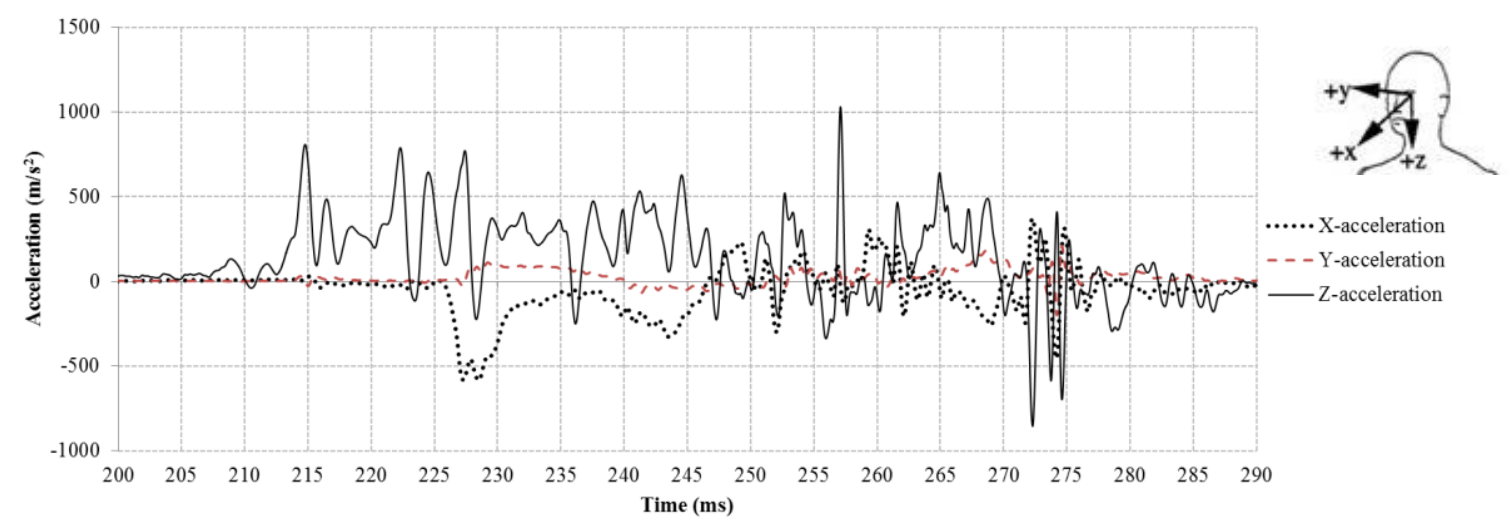

Figure 11. Component acceleration data of child head dummy at 200-290 ms (MCT0120).

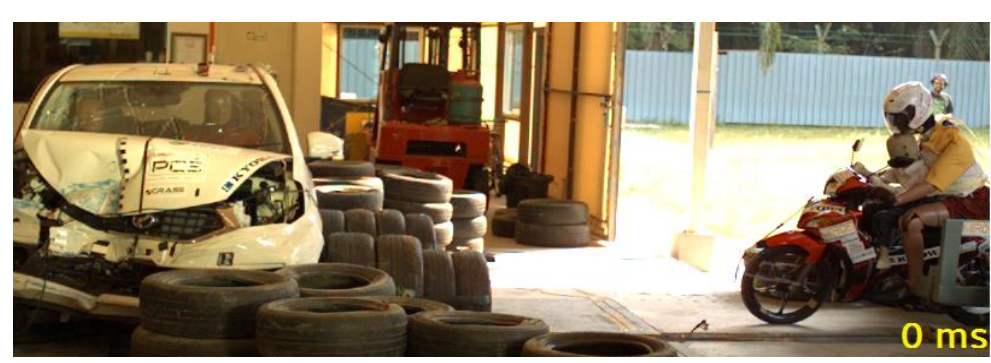

(a)

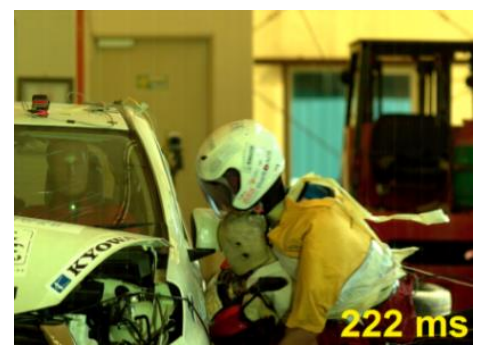

(c)

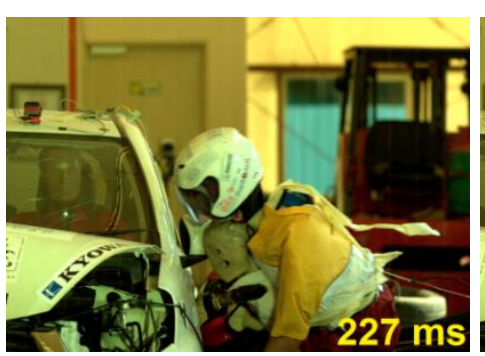

(d)

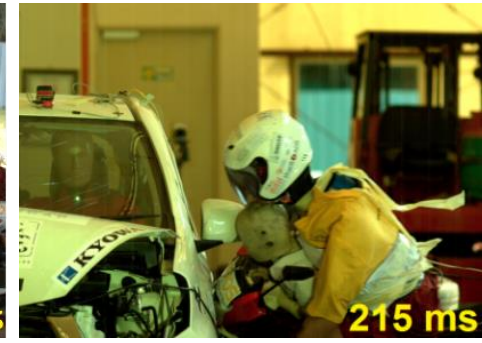

(b)

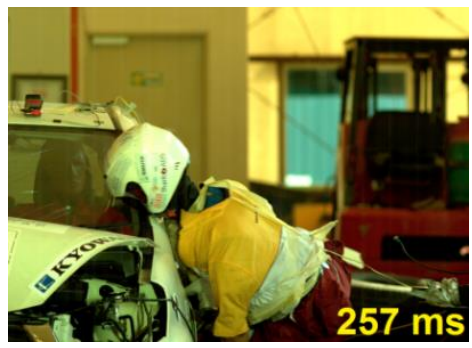

(e) 


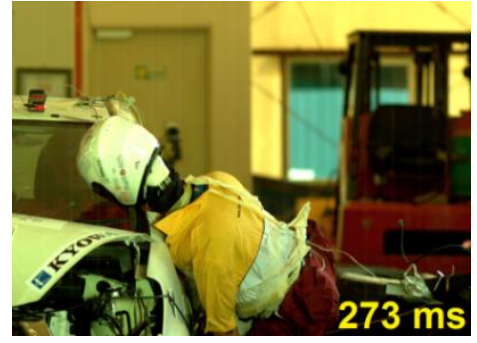

(f)

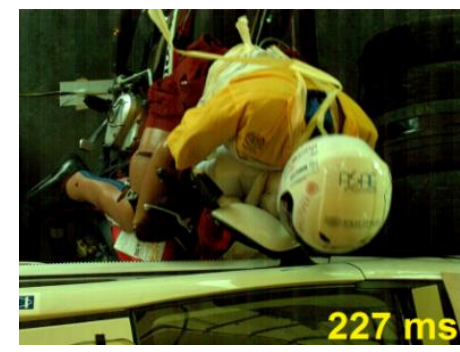

(i)

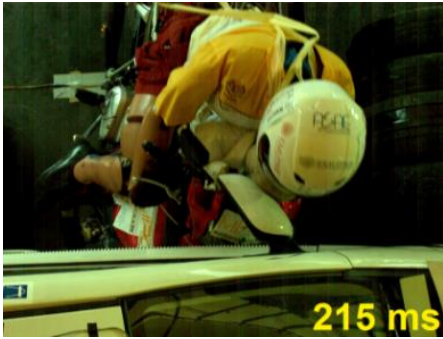

$(\mathrm{g})$

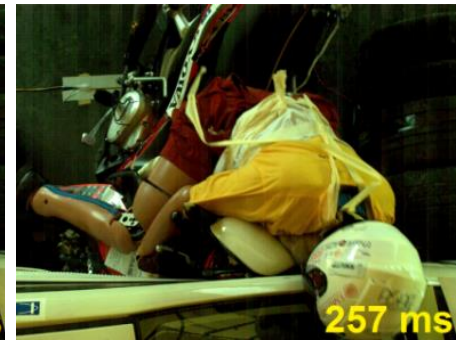

(j)

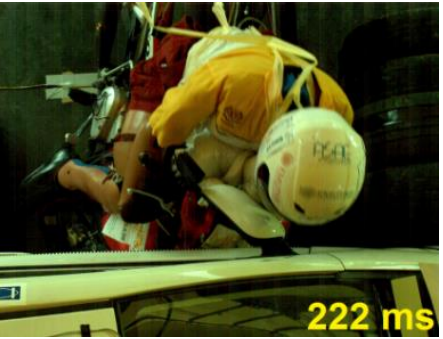

(h)

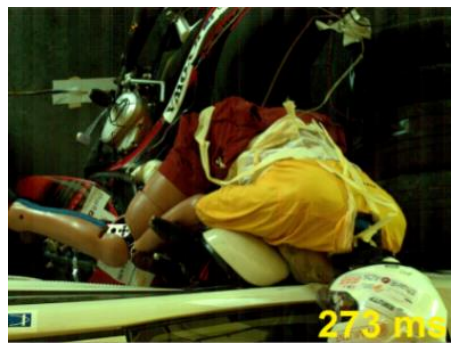

(k)

Figure 12. Kinematics of dummies (MCT0120) for far-side view at (a) $0 \mathrm{~ms}$, for nearside view at (b) $215 \mathrm{~ms}$, (c) $222 \mathrm{~ms}$, (d) $227 \mathrm{~ms}$, (e) $257 \mathrm{~ms}$ and; (f) $273 \mathrm{~ms}$, and for top view at (g) $215 \mathrm{~ms}$, (h) $222 \mathrm{~ms}$, (i) $227 \mathrm{~ms}$, (j) $257 \mathrm{~ms}$ and; (k) $273 \mathrm{~ms}$.

\section{DISCUSSION}

The data from the high-speed camera reveal two different kinematics of the child pillion passenger from the two crash tests. From both tests, the lifting up of the rear wheel during impact are observed. During the impact, the part on motorcycle front wheel which impacted to the car acted like a pivot point in the longitudinal direction. The weight of the MC and occupants results in the anticlockwise moment about the pivot. While the inertial force acting at the Center of Gravity of the MC-occupants system results in the clockwise rotation about the pivot. The inertial force leads to the raising of rear wheel if the deceleration is high. This phenomenon can also be observed during the motorcycle pitch over brake application.

For the first test (child sitting behind the rider), the lifting up of rear wheel leads to the ejection of the rider and the child passenger from the MC. The child passenger lied on the rider during impact. Once the rider head impacted the car, the child was still moving along the rider back. The rider can then be considered as a launching ramp for the child passenger. However, in this first test the leg of rider was dragged by the stoppers before the impact which made the MC tilted. The child head then impacted to the A-pillar. However, the child head impact location may alter a bit if the MC did not tilt. The highest peak is observed when the head of child hit the A-pillar of the car. The secondary ground impact occurring later has much lower peak. The corresponding $\mathrm{HIC}_{15}$ is above the threshold of 650 implying the severe head injury. This situation is similar to the rider trajectory condition under the pitch-over brake applications and impacts [16]. Furthermore, the head impact of child dummy can be used as one of four approaches to modify road user behavior through the increment of road user's awareness of risk for child pillion in motorcycle [17]. Therefore, the use of helmet can reduce the HIC values and accelerations experienced by child's head as suggested by Word Health Organization [5]. However, head injury for young children is distinct from 
that for adult head because of mechanisms and injury thresholds [18]. If the child helmet is developed by numerical simulation with the head injury criteria for adult head, the injury protection of child head could not be effective due to different mechanical properties. To investigate child head injury, the statistically based neurological injury criteria for pediatric population using finite element analysis is proposed [19].

However, for the second test (child sitting before the rider), the chest and neck of child dummy was compressed by the adult dummy against the car body because of in-line kinematics motion of both dummies. The child head impacted after his torso with lower peak of acceleration than in the first test. HIC value is lower than the threshold value of 650 . This implies very low risk of head injury. The child thorax and neck injuries are more pronounced for this second test. The lifting of rear wheel does not affect the kinematics of child passenger in the case of child sitting in front of the rider. This is because the child sitting position is close to the front wheel. As the child sits between the rider's arms, secondary ground impact is not seen. These results can support the concept ideal suggestion of child seat with restraint system and spoke protection for child's foot as minimally-acceptable safety [5]. To develop such child seat in motorcycle, the experimental data from the motorcycle crash test with child dummy can be used in finite element analysis technique with consideration of the chest injury criteria.

A limitation of this work is the number of experiments which are not enough to show repeatability. However, the objective of this work is to study the kinematics and potential injury mechanisms of the child pillion passenger sitting at two different locations. The experiments done are enough to show two different kinematics and injury mechanisms obtaining from child sitting behind the rider and before the rider. The experimental results of this work can be subsequently used for numerical model validations. The validated multibody models or finite element models can be employed for further investigation of child pillion passenger kinematics and injury mechanisms at various impact speeds and impact angles.

\section{CONCLUSION}

Motorcycle crash tests with two sitting locations of child pillion passenger were completed. Two significantly different global kinematics and injury mechanisms of the child pillion passenger are observed. Both crash tests show the raising of the MC rear wheel during the impact. This phenomenon has influence on the kinematics of child passenger sitting behind the rider. The child passenger was thrown over the rider back. The child head then impacted to the A-Pillar leading to high risk of severe head injury. However, the raising of rear wheel has less effect on the child sitting in front of the rider close to the front wheel. In this test, the child passenger impacted the car structure before the rider. The child's back and neck was impacted by the rider torso leading to high risk of severe thorax compression and cervical spine injury. Low risk of severe head injury is seen in this case since the trunk of the child impacted the vehicle structure and already absorbed amount of impact energy before the head came to impact. The child passenger's thorax and neck injuries due to the rider impact from behind are difficult to protect with existing protective gears. However, with available helmets and child seats, the child sitting behind the rider will be safer as the severe head injury can be mitigated using this protective equipment. The experimental data obtained from these two tests can be subsequently employed for validation of numerical models. These 
models can be used for further investigation of kinematics and injury mechanisms of child pillion passenger for various impact speeds, impact location and impact angles.

\section{ACKNOWLEDGEMENT}

The authors would like to thank Thairoad and Thai public health foundation for financial support and special thanks to Malaysia Institute of Road Safety Research for technical information and laboratory facilities.

\section{REFERENCES}

[1] Lin MR, Kraus JF. A review of risk factors and patterns of motorcycle injuries. Accident Analysis Prevention 2009; 41(4):710-722.

[2] World Health Organization, Global status report on road safety 2015: supporting a decade of action. Geneva, Switzerland: WHO, 2015.

[3] Berg FA, Rücker P, König J. Motorcycle crash tests - an overview. International Journal of Crashworthiness 2005; 10(4):327-339.

[4] Berg FA, Rucker P, Gartner M, Konig J, Grzebieta R, Zou R. Motorcycle impacts into roadside barriers-Real-world accident studies, crash tests and simulations carried out in Germany and Australia. In: Proceedings of the Nineteenth International Conference on Enhanced Safety of Vehicles, Washington DC, USA 2005.

[5] World Health Organization. Child development and motorcycle safety. Retrived from http://apps.who.int/iris/bitstream/10665/173782/1/9789290224860DPR.pdf?ua=1: 2015.

[6] Paiman NF, Mi Z, Aqbal HA. Safety helmet use rate and child pillion riders' characteristics in Selangor.Malaysian Institute of Road Safety Research reserch report 2013.

[7] Oxley J, Ravi MD, Yuen J, Hoareau E, Hashim H. Identifying contributing factors to fatal and serious injury motorcycle collisions involving children in Malaysia. Annual Advance Automotive Medicine Associate Advance Automotive Medicine Annual Science. Confonference. 2013;7, 329-336.

[8] Tan CL, Wong SV. Motorcycle crash test center: a movable barrier approach. International Journal of Automotive Mechanical Engineering. 2012; 5: 630-638.

[9] Serre T, Masson C, Perrin C, Martin JL, Moskal A, Llari M. The motorcyclist impact against a light vehicle: Epidemiological, accidentological and biomechanic analysis. Accident Analysis Prevention. 2012; 49: 223-228.

[10] Kasantikul V, Ouellet JV, Smith T, Sirathranont J, Panichabhongse V. The role of alcohol in Thailand motorcycle crashes. Accident Analysis Prevention.2005;37(2):357-366.

[11] International Organization for Standardization. ISO-13232-6---Motorcycles Test and analysis procedures for research evaluation of rider crash protective devices fitted to motorcycles - Part 6: Full-scale impact test procedures. 2005.

[12] The United Nations Economic Commission for Europe (UNECE). Regulation No. 94 Uniform provisions concerning the approval of vehicles with regard to the protection of the occupants in the event of a frontal collision. Retrieved from: $\quad$ http://www.unece.org/trans/main/wp29/wp29regs81-100.html: 9 September 2015. 
[13] The European New Car Assessment Programme. Protocols- Pedestrian Protection. Retrived from https://www.euroncap.com:443/en/vehicle-safety/theratings-explained/ pedestrian-protection/ : 04 January 2018.

[14] Lagenda 115Z - Product Details - Hong Leong Yamaha. Retrived from http://www.yamaha-motor.com.my/products-details.php?id=5; 25 February 2016.

[15] Perodua Myvi. Retrived from http://www.perodua.com.my/ourcars/myvi: 25 Febuary 2016.

[16] Frank TA, Smith JW, Hansen DC, Werner SM. Motorcycle rider trajectory in pitch-over brake applications and impacts. SAE Technical Paper, 2008.

[17] Rothengatter T. Psychological aspects of road user behaviour. Journal of Applied Psychology; 46 (3): 223-234.

[18] Duhaime AC, Alario AJ, Lewander WJ. Head Injury in Very Young Children: Mechanisms, Injury Types, and Ophthalmologic Findings in 100 Hospitalized Patients Younger Than 2 Years of Age. Pediatrics 1992; 90:179-185.

[19] Roth S, Vappou J, Raul JS, Willinger R. Child head injury criteria investigation through numerical simulation of real-world trauma. Computer Methods Programs Biomedical. 2009; 93(1): 32-45. 